\title{
A multi-zone biomonitoring buffer concept for protected areas to conserve biological di- versity and prevent adverse effects of anthropogenic pollution
}

\author{
Samuel J. Biondo
}

Keywords: biomonitoring, buffer zones, protected areas, biological diversity, habitat protection

\section{Abstract}

This paper describes a concept for combining the biomonitoring ability of plants with their capacity to block the transport of pollutants before they can contribute to habitat degradation. Biomonitoring and buffer zones have not yet been combined to create an effective biological system for detecting, monitoring and limiting the transport of pollution from anthropogenic sources to conserve biological diversity and prevent adverse effects. This paper presents a multi-zone biomonitoring buffer concept to achieve those goals.

\section{Biomonitoring}

Biomonitoring has been practiced for over 100 years (Nylander 1886). Biomonitoring can be used to detect and monitor the temporal, spatial and cumulative effects of different pollutants in the ecosystem and to determine the potential for long-term harmful effects (De Temmerman et al. 2005). A very wide range of species and plant types have been studied as potential monitoring agents. Lichens, fungi, tree bark and leaves of higher plants are commonly used to detect the deposition, accumulation and distribution of pollutants in the environment. Sensitive plants may show visible effects of pollution long before their effects can be observed on animals or materials.

Plants have been used to establish field monitoring networks in Europe, Canada and the United States. One example is the assessment of the effects of ozone and atmospheric heavy metal deposition conducted through the pan-European biomonitoring programme operating according to a common protocol, within the framework of the International Cooperative Programme on Effects of Air Pollution on Natural Vegetation and Crops under the Convention on Long-Range Transboundary Air Pollution (ICP Vegetation 2013).

Biomonitoring has many advantages but it requires sophisticated applications. Cape $(2009,2012)$ discusses when and when not to use plants as bioindicators, and illustrates some of the precautions required if meaningful conclusions are to be inferred. He notes that the sound interpretation of measurement data relies on a clear understanding of what such biomonitors can and cannot demonstrate, and the limitations of each approach.

De Temmerman et al. (2004) report that lack of standardization is probably one of the major reasons why biomonitoring techniques are less used in legislation than methods based on physico-chemical monitoring. They noted, however, that both techniques are complementary because physico-chemical monitors measure pollutant concentrations or deposition fluxes, whereas biomonitors reflect effects.
The use of indicator plants may obviate the necessity for expensive equipment but successful applications require tailored analytical plant studies compared to using commercially available physico-chemical monitoring instruments that are applied routinely in a cookbook fashion.

\section{Buffer zones}

Various types of buffer zones have been implemented for protective areas for several decades. The term buffer zone first appears to have become widely used with the Man and the Biosphere programme, and the biosphere reserves in the 1970s, which aimed to set a scientific basis for the improvement of the relationships between people and their environment globally (Martino 2001). Buffer zones are often created to enhance the protection of a conservation area. Buffers are commonly used in a variety of social functions, in addition to attempting to control air or water quality (Benthrup 2008).

Protected areas are supposed to be safe havens. However, at the present time protected areas are generally not considered islands safe from negative external effects, which can have serious impacts on species and habitats within them (Pan-European Ecological Networks 2013). Shafer (1999) prophetically noted that the science of buffer zones is very immature and deserves more attention and stated that a comprehensive, overall protected area strategy must include more than just buffer zones. Some authors (Shafer 1999; Bentrup 2008; Mehring \& Stoll-Kleemann 2011) have already pointed out that buffer zones can remedy some impacts but not others and social obstacles can further limit their effectiveness.

Although there is a substantial literature base, there are no manuals for designing buffers. A large body of scientific knowledge exists to help guide the planning and designing of buffers. This information is widely dispersed throughout the vast research literature and is not easily accessible or usable for most planners (Bentrup 2008). Clearly, buffer zones have not been designed to guarantee the same level of protection 


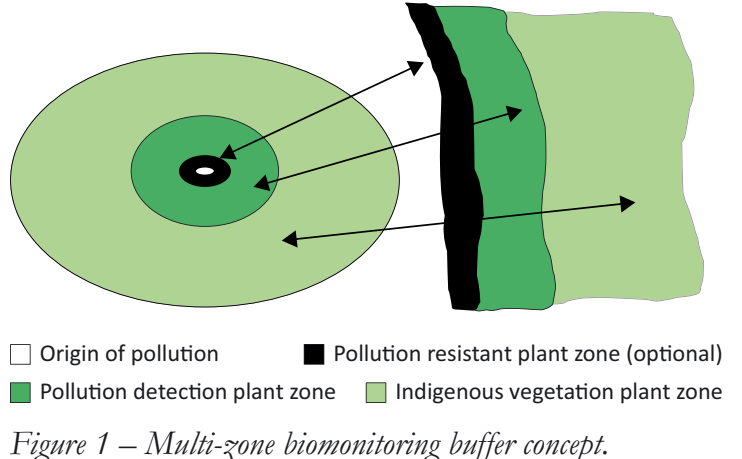

expected of buffers used in chemistry laboratories, where buffers are expected to maintain the original condition $(\mathrm{pH})$. However, buffer zones could be designed to take better advantage of the natural ability of certain plants to bioaccumulate, degrade or render harmless contaminants in the air, water or soil.

\section{Multi-zone biomonitoring buffer concept}

Figure 1 introduces a multi-zone concept combining biomonitoring with buffering and expanding the function of buffer zones to block the transport of pollution from external sources. The origin of pollution from point sources is represented in the centre of concentric ellipses and pollution from non-point sources, including, for instance, transportation corridors, originates at the inside edge of the linear / curvilinear strips. An optional pollution resistant plant zone could serve as the first protective barrier, depending upon trade-offs between the benefits of pollution resistance and possibly undesirable characteristics of tolerant varieties or some non-native plants. Invasive plants would be excluded because they can alter habitats and reduce biodiversity by choking out other plant life, putting pressure on native plants and animals, including threatened species that may succumb. The pollution detection plant zone can be a series of subzones, including, say, two layers, each of which might be comprised of sublayers of different types of vegetation. Legal permits for anthropogenic activities could possibly allow pollution excursions to penetrate the first layer of the pollution detection plant zone, which could be comprised of somewhat resistant plants, but not allow pollution to occur in the very sensitive plants comprising the next sublayer within this zone. Hence, the first two zones would serve to protect the indigenous vegetation zone from pollution damage. Carefully designed plant studies will be required to set up the pollution detection plant zone. In addition to forests and urban forests, bodies of water, such as bays, canals, channels, falls, gulfs, lakes, rivers and straits - that are not sources of pollution - could possibly benefit from the first two zones.

Multi-tier buffer concepts are not new. For example, multi-tier buffers are used for riparian management (Parkyn 2004) with vegetated buffer zones, but the strips of land that separate upland or hillslope areas from streams, lakes or wetlands (riparian buffers) are managed for the purpose of removing pollutants from runoff or groundwater and they are not designed with provisions for biomonitoring.

The multi-zone concept should enhance the ecological network concept that was developed in Europe to counteract physical fragmentation, which jeopardizes the viability of ecosystems and species populations (Bennett \& Mulongoy 2006). Although the ecological network concept was primarily created for rural areas, it has also been studied for application in urbanized areas. A diagram of the ecological network concept is shown in Figure 2. The exploded view at the top left shows the modification of the traditional buffer zone with the addition of pollution resistant and pollution detection layers.

The core areas are the protected areas, which are connected by corridors that allow the movement of animal and plant species between the core areas, and both are surrounded by buffer zones. The corridors can include long, uninterrupted strips of vegetation, which are termed linear corridors; stepping stone corridors, which are small, non-connected habitats used to find shelter, food or for rest; and landscape corridors which are strips of habitat that connect isolated patches of habitat. Substituting the multi-layer buffer zones for the weaker buffer zones in the ecological network model could stop negative external effects, such as air or water pollution, on protected areas, which can have serious impacts on species and habitats within them.

The multi-zone concept should also enhance the greenways concept (White 1959) that was developed in the United States. The term greenways refers to a system of interconnected linear territories that are protected, managed and developed so as to obtain ecological, recreational, historical and cultural benefits, or a system of routes dedicated to non-motorized traffic connecting people with landscape resources (natural, agricultural, historical-cultural) and the centres of life (public offices, sport and recreational facilities, etc.) both in the urban areas and in the countryside (Toccoloni et al. 2006).

Greenways are frequently corridors within an urban, suburban or rural context, slotted into an artificial landscape. Greenways generally develop along linear structures or elements already present in the surrounding landscape, such as natural corridors (rivers, valleys and ridges), disused railways, canals and embankments, and panoramic roads or minor rural roads (Little 1990). Therefore they offer the advantage of being easily established even in areas that are critical in terms of competition for space. Both ecological networks and greenways are linear structures crossing the landscape, both perform a connecting function in that they are elements created for migration and movement (in one case of flora and fauna and in the other of humans) and both generally contain vegetation (Fumagalli \& Toccolini 2012). 


\section{Discussion}

The multi-zone biomonitoring buffer concept is essentially a biological filtering system with inherent pollution detection and monitoring capabilities. Its applications are not limited to ecological networks. It can also enhance the numerous other conventional vegetative buffer concepts, e.g. riparian buffers. It can permit easy sampling and long-term monitoring without the need for expensive equipment. It does not require new technology or exotic materials to be developed to test its implementation. It is a novel concept, combining proven elements, that has not been discussed or reported in the literature. There are several possible reasons why this concept has not yet been engineered effectively into a useful system. The most likely include:

- Resistance to creating or expanding buffers for protected areas due to the politics of land use in buffer zone communities. Bennett \& Mulongoy (2006) concluded that although the concept of a buffer zone may be straightforward, its design and its functioning in practice can raise many challenges. Adequately understanding the interaction between human activities and species populations and the resulting dynamics is a complex issue; determining appropriate land uses is therefore far from easy. Decisions to restrict human activities in buffer zones will also impose costs on the landowners and users, raising the question of compensation.

- Historically ineffective permit systems for siting and operating industrial facilities or regulating effluents from human activities; permit systems for siting and operating point source facilities and for (non-point source) diffuse sources of pollution are known to be frequently ineffective due to loopholes, enforcement failures and other flaws (Collins 2010).

- The sophisticated nature of plant studies. It has been said that biomonitoring could appear to some observers to be the domain of do-it-yourself $\mathrm{PhD}$ scientists who spent years studying its intricacies. Choosing the plants for the pollution resistant and pollution detection zones multi-zone biomonitoring buffer concept will require carefully designed pilot studies but no new technology is required.

\section{Future directions}

Testing the multi-zone biomonitoring buffer concept could occur through pilot studies conducted at various sites and scales. Laboratory and / or additional field investigations may be necessary to determine the role of individual pollutants, the synergistic effects of pollutant mixtures, biological responses and tolerances. These studies can be used to establish parameters of biological monitoring programmes conducted under natural conditions. The pilot study initiated here could be considered a jumping off point for a long-

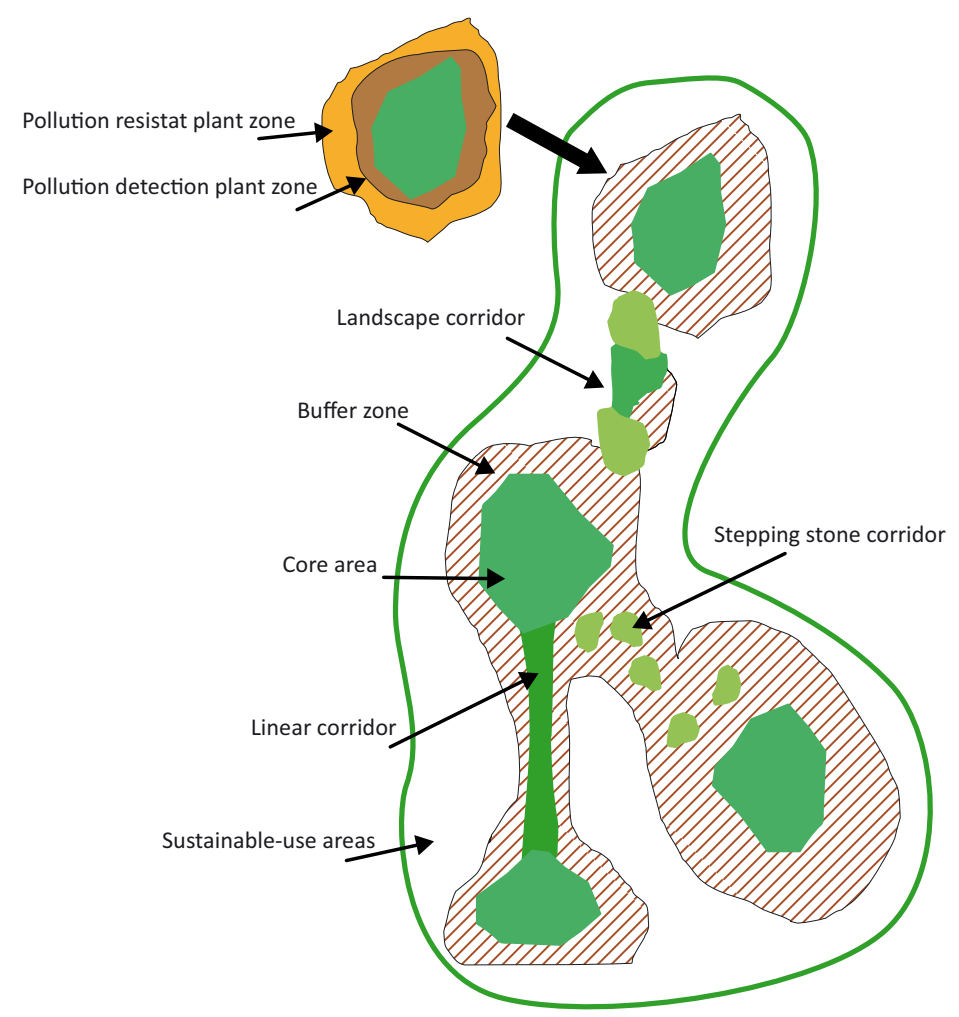

Figure 2 - Diagrammatic representation of the spatial arrangement for an ecological network (after Bennett \& Mulongoy 2006). The exploded view at the top left shows the modification of the traditional buffer rone with the addition of pollution resistant and pollution detection layers.

term study to continue to validate and refine the concept.

\section{Conclusion}

Human activities have increasingly contributed to habitat destruction, degradation and fragmentation. Effective habitat management and maintenance measures are needed to reverse this trend, including coherent and comprehensive environmental control and management systems, sufficient financial and technological support, transparent effective permitting systems, and effective enforcement. This paper describes a concept for combining the biomonitoring ability of plants with their capacity to block the transport of pollutants before they can contribute to habitat degradation. This multi-zone biomonitoring buffer concept, when implemented through an effective permit system, can contribute to preventing damage and providing stability to protected areas.

\section{References}

Bennett, G. \& K.J. Mulongoy 2006. Review of Experience with Ecological Networks, Corridors, and Buffer Zones. CBD Technical Series No. 23. Secretariat of the Convention on Biological Diversity.

Bentrup, G. 2008. Conservation Buffers-Design Guidelines for Buffers, Corridors, and Greenways. U. S. Depart- 
ment of Agriculture, Forest Service, Southern Research Station. Ashville, N.C.

Cape, J.N. 2009. Plants as Accumulators of Atmospheric Emissions. In: Legge, A.H. (ed.), Relating Atmospheric Source Apportionment to Vegetation Effects: Establishing Cause and Effect Relationships. Developments in Environmental Science 9: 61-97.

Cape, J.N. 2012. Plants as accumulating biomonitors. In: Samson, R. et al., Biomonitoring of air quality with plants, animals and humans. BIOMAQ Conference November 12-14 2012, Antwerp, Belgium. Book of abstracts: 5-6.

Collins, C. 2010. Toxic Loopholes: Failures and Future Prospects for Environmental Law. Cambridge.

De Temmerman, L., J.N.B. Bell, J.P. Garrec, A. Klumpp, G.H.M. Krause \& A.E.G. Tonneijck 2004. Biomonitoring of air pollutants with plants - considerations for the future. In: Klumpp, A., W. Ansel \& G. Klumpp (eds.), Urban Air Pollution, Bioindication and Environmental Awareness. EuroBionet 2002 Conference on Urban Air Pollution, Bioindication and Environmental Awareness in Hohenheim 05.11.2002. 337-373.

De Temmerman, L., J.N.B. Bell, J.P. Garrec, A. Klumpp, G.H.M. Krause \& A.E.G. Tonneijck 2005. Biomonitoring of Air Pollutants with Plants. EnviroNews 11 (2).

Fumagalli, N. \& A. Toccolini 2012. Relationship Between Greenways and Ecological Network: A Case Study in Italy. International Journal of Environmental Research 6 (4): 903-916.

ICP Vegetation 2013. Available at: http:/ /icpvegetation.ceh.ac.uk/ (accessed on: 01/06/2013)

Little, C.E. 1990. Greenways for America. Baltimore.

Martino, D. 2001. Buffer Zones Around Protected Areas: A brief Literature Review. Electronic Green Journal 1 (15).
Mehring, M. \& S. Stoll-Kleemann 2011. How effective is the buffer zone? Linking institutional processes with satellite images from a case study in the Lore Lindu Forest Biosphere Reserve, Indonesia. Ecology and Society 16 (4): 3.

Nylander, W. 1886. Les lichens du Jardin du Luxembourg. Bulletin de la Societé Botanique de France 13: 364-372.

Pan-European Ecological Networks 2013. IUCN Countdown 2010. Available at: http://www.countdown2010.net/archive/paneuropean.html (accessed on: 01/06/2013)

Parkyn, S. 2004. Review of Riparian Buffer Zone Effectiveness. MAF Technical Paper No: 2004/05: 2.

Shafer, C.L. 1999. U S National Park Buffer Zones: Historical, Scientific, Social, and Legal Aspects. Environmental Management 23 (1): 49-73.

Toccolini, A., N. Fumagalli \& G. Senes 2006. Greenways planning in Italy: the Lambro River Valley Greenways System. Landscape and Urban Planning 76: 98-111.

White, W.H. 1959. Securing Open Space for Urban America: Conservation Easements. Urban Land Institute - Technical Bulletin 36. Washington.

\section{Author}

\section{Samuel J. Biondo}

is an independent consultant with a business practice focused on energy and environmental technology. He advises county, state and federal government agencies on energy, air and water quality issues. $\mathrm{He}$ earned undergraduate degrees from The Pennsylvania State University and Johns Hopkins University and graduate degrees from George Washington University. He can be reached at 1-301-602-4655. Contact: sjbiondo@verizon.net 